$\mathrm{p}=0.003$ ) and regional parameters (data for 12 segments; vs $10.8 \pm 4.4$ vs $9.9 \pm 4.4 \mathrm{~cm} / \mathrm{s}, \mathrm{p}<0.0001 ; \mathrm{S}-15.7 \pm 4.7$ vs $-14.8 \pm 4.4 \%$, $\mathrm{p}=0.03$; SR $-2.2 \pm 0.5 \mathrm{vs}-2.0 \pm 0.5 / \mathrm{s}, \mathrm{p}<0.0001)$. The improvement was greater in those regions subtended by a coronary artery with $>50 \%$ stenosis than in those supplied by a non-obstructed artery. At 30 min recovery, ischaemic LV dysfunction did not occur after sitagliptin (LVEF $55.6 \pm 3.5$ vs $49.7 \pm 2.6 \%, \quad \mathrm{p}=0.0001 ; \mathrm{MASV}$ $5.55 \pm 1.2$ vs $5.20 \pm 1.0 \mathrm{~cm} / \mathrm{s}, \mathrm{p}=0.003)$.

Conclusion In patients with CAD and T2DM receiving OHT, DPP-4 inhibition by sitagliptin improves both global and regional myocardial performance during demand ischaemia (with greater benefit seen in ischaemic vs non-ischaemic segments), and mitigates post-ischaemic stunning.

\section{INCREASE IN LEFT VENTRICULAR MASS IN TYPE 2 DIABETES IS DEPENDENT ON DURATION OF DIABETES}

doi:10.1136/heartjnl-2012-301877b.136

${ }^{1}$ A K Ghosh, ${ }^{* 1}$ A D Hughes, ${ }^{1} \mathrm{~N}$ Chaturvedi, ${ }^{1} \mathrm{D}$ P Francis, ${ }^{2} \mathrm{D}$ Pellerin, ${ }^{3} \mathrm{~J}$ Deanfield, ${ }^{4} \mathrm{M}$ Pierce, ${ }^{4} \mathrm{D}$ Kuh, ${ }^{1} \mathrm{~J}$ Mayet, ${ }^{4} \mathrm{R}$ J Hardy. ${ }^{1}$ International Centre for Circulatory Health, Imperial College, London, UK; ${ }^{2}$ The Heart Hospital, London, UK; ${ }^{3}$ Institute of Child Health, University College London, London, UK; ${ }^{4}$ Medical Research Council Unit for Lifelong Health and Ageing, London, UK

Background Adverse consequences of type 2 diabetes mellitus (T2DM) include an increase in left ventricular mass (LVM). Whether the underlying mechanisms of this association are due to the hyperglycaemic state per se, or to other risk factors is unclear. We explored the association between diabetes duration, an index of the chronicity of the hyperglycaemic state, with LVM.

Methods The Medical Research Council National Survey of Health and Development is a birth cohort of men and women born in Britain in 1 week in March 1946. When study members were 60-64 years of age, 1700 underwent echocardiography according to ASE guidelines and LVM indexed to height 2.7 (LVMI) was calculated. Date of diagnosis of T2DM by a doctor was obtained in the 175 cohort members reporting T2DM through information supplied on postal questionnaires and at interview. Linear regression models were fitted to estimate the effects of presence and of duration of diabetes on LVMI at age 60-64 years.

Results In a linear regression model adjusted for sex, current body mass index (BMI), and current systolic blood pressure (SBP), LVMI increased by $0.78 \mathrm{~g} / \mathrm{m}^{2}$ for every year increase in diabetes duration. When diabetes duration was divided into categories, this same trend was observed. Compared with those with no diagnosis of diabetes, LVMI was $3.82 \mathrm{~g} / \mathrm{m}^{2}$ (95\% CI 0.73 to $\left.6.93, \mathrm{p}=0.02\right)$ higher for $0-10$ years duration of diabetes, $7.46 \mathrm{~g} / \mathrm{m}^{2}(95 \% \mathrm{CI}-1.94$ to 16.87 , $\mathrm{p}=0.12)$ higher for $10-20$ years duration, and $17.28 \mathrm{~g} / \mathrm{m}^{2}(95 \%$ CI 1.01 to 33.56, $\mathrm{p}=0.04$ ) higher for $20-30 \mathrm{y}$ duration of diabetes.

Conclusions LVMI increases significantly with diabetes duration, even when other major risk factors are accounted for. As T2DM is increasingly being diagnosed at younger ages, this duration effect has important implications for the future burden of associated cardiac disease, and indicates that hyperglycaemia per se plays a key role in the adverse consequences of T2DM on the left ventricle. This also has implications for current treatment and prevention strategies.

\section{7 \\ TIME FOR A REVIEW OF THE "WATCH AND WAIT" STRATEGY FOR YOUNG BORDERLINE-HYPERTENSIVES?}

doi:10.1136/heartjnl-2012-301877b.137

${ }^{1} \mathrm{~A}$ K Ghosh, ${ }^{*} \mathrm{R}$ J Hardy, ${ }^{1} \mathrm{D}$ P Francis, ${ }^{1} \mathrm{~N}$ Chaturvedi, ${ }^{3} \mathrm{D}$ Pellerin, ${ }^{4} \mathrm{~J}$ Deanfield, ${ }^{2} \mathrm{D}$ Kuh, ${ }^{1} \mathrm{~J}$ Mayet, ${ }^{1} \mathrm{~A} D$ Hughes. ${ }^{1}$ International Centre for Circulatory Health, Imperial
College, London, UK; ${ }^{2}$ Medical Research Council Unit for Lifelong Health and Ageing, London, UK; ${ }^{3}$ The Heart Hospital, London, UK; ${ }^{4}$ Institute of Child Health, University College London, London, UK

Background In cross-sectional studies, elevated systolic blood pressure (SBP) is associated with increased left ventricular mass (LVM), which leads to increased cardiovascular morbidity and mortality. Current guidelines recommend a "watch and wait" approach in younger individuals with a borderline-high SBP. We investigated if this was a safe assumption.

Methods The Medical Research Council National Survey of Health and Development is a birth cohort study following men and women born in Britain in 1 week in March 1946. When study members were 60-64 years of age, 1700 underwent echocardiography and LVM indexed to body-surface-area (LVMI) was measured. The relationship between repeated measures of SBP and antihypertensive treatment (measured at 4 time points: ages 60-64 (current), 53, 43 and 36) and LVMI at 60-64 years was examined using sex-adjusted multiple regression models. Then, multilevel models of SBP were used to estimate person-specific intercepts (SBP at age 36) and slopes (rate of change in SBP between 36 and 53 years). The intercepts and slopes were then included in sex-adjusted linear regression models with LVMI as the outcome.

Results Individuals on treatment for hypertension, from age 43 years onwards, had higher mean LVMI than those who were not on treatment, irrespective of level of SBP at the same age. LVMI was $12.3 \mathrm{~g} / \mathrm{cm}^{2}$ (95\% CI 9.2 to $15.4 ; \mathrm{p}<0.001$ ) higher for those currently on treatment, $10.0 \mathrm{~g} / \mathrm{cm}^{2}$ higher (95\% CI 5.9 to $14.1 ; \mathrm{p}<0.001$ ) for those on treatment at age 53 , and $15.1 \mathrm{~g} / \mathrm{cm}^{2}$ higher $(95 \%$ CI 5.8 to 24.3; $\mathrm{p}=0.001$ ) for treatment at age 43 . In associated analyses, the effect of mid-life rate of change in SBP (from 36 to 53 years) on LVMI at age 60-64 years was 10 times greater than the effect of more recent rate of change in SBP (from 53 years to current).

Conclusions Our research suggests being on antihypertensive treatment may not normalise LVMI due to irreversible cardiac damage occurring in mid-life in poorly controlled hypertensives. Early identification and effective treatment of individuals with rapidly increasing SBP in mid-life may be key to preventing such damage. A review of current guidelines on monitoring and screening of blood pressure may thus be required.

\section{NON-ANGINAL CHEST PAIN: NOT AS BENIGN AS WE WOULD LIKE TO BELIEVE!}

doi:10.1136/heartjnl-2012-301877b.138

A George, ${ }^{*}$ T Anwar, K Advani, D Cullington, J John, S Chattopadhyay. Scunthorpe General Hospital, Scunthorpe, UK

Background In the Quick Reference Guide of the clinical guidance 95 (CG95) NICE recommends that chest pain (CP) diagnosed as nonanginal (NA) should not be investigated for stable angina routinely. In the Full Guidance, it qualifies this statement suggesting that stable angina should be excluded in patients with NACP unless clinical suspicion is raised based on other aspects of the history and risk factors. In the chest pain management algorithm, however, it excludes patients with NACP in whom stable angina is suspected based on history and risk factors. This study was undertaken to assess the outcome of patients attending rapid access chest pain clinic (RACPC) and diagnosed with NACP who are likely to be discharged without further investigation as suggested by CG95.

Method and Results 1042 consecutive RACPC referrals between November 2009 and April 2011 were reviewed. Demographics, CG95 defined risk factors, CP characteristics, history of confirmed coronary artery disease (CAD), results of the exercise ECG test, management plan and outcomes (composite end point of all cause 
mortality, non-fatal myocardial infarction, referral/admission to hospital with CP) up to a minimum of 6 months after the index assessment were analysed. Typical, atypical and NACP were determined from CP characteristics. 622 patients without confirmed CAD were diagnosed with NACP. Following the 1st visit, 70 patients were recommended coronary angiograms (27 significant CAD, (26 revascularised, 1 treated medically), 7 refused) and 66 had myocardial perfusion scans (14 reversible/2 fixed defects). 62 (10\%) patients reached an end-point (2 cancer deaths, 11 non-fatal MI, 40 readmitted and 8 rereferred with CP, 1 nonhaemorrhagic stroke). Of these patients, 29 were previously investigated: 21 angiograms (10 significant CAD), 14 MPS (3 reversible/1 fixed defect). Investigations after reaching end-point showed 3 significant CAD (14 angiograms) and 1 reversible defect (5 myocardial perfsion scan). 14 others were not investigated further due to unequivocally negative exercise ECG. Only the presence of diabetes predicted the end-point (OR 5.21, 95\% CI 2.67 to $10.15, p<0.0001)$ in a multiple logistic regression analysis using age, gender, current smoking, total cholesterol $>6.47 \mathrm{mmol} / \mathrm{l}$ and diabetes as covariates.

Conclusion $47(7.6 \%)$ patient that are to be discharged without investigation, had significant coronary artery disease. Even with a short follow-up, $10 \%$ of these patients needed medical attention for suspected cardiovascular morbidity. It may be unreasonable to discharge patients describing NACP especially diabetics.

\section{CLINICAL OUTCOMES OF PATIENTS EXCLUDED FROM CARDIAC INVESTIGATION IN THE NICE GUIDELINES FOR CHEST PAIN OF RECENT ONSET}

doi:10.1136/heartjnl-2012-301877b.139

${ }^{1} \mathrm{C}$ Patterson, ${ }^{* 2} \mathrm{~N}$ Ahmed, ${ }^{3} \mathrm{E}$ Nicol, ${ }^{4} \mathrm{~L}$ Bryan, ${ }^{1} \mathrm{D}$ Bell. ${ }^{1}$ Imperial College, London, UK; ${ }^{2}$ Ealing Hospital, London, UK; ${ }^{3}$ Royal Brompton Hospital, London, UK; ${ }^{4}$ St Thomas' Hospital, London, UK

Introduction NICE guidance for the investigation of chest pain of recent onset ${ }^{1}$ recommends clinical assessment and risk stratification within a diagnostic algorithm. Patients with pain considered "nonanginal", and those with atypical/typical anginal pain but a likelihood of coronary artery disease $(\mathrm{CAD})<10 \%$ are not routinely recommended for cardiac investigation. This study sought to assess whether clinical outcomes support these patients being considered at low risk of CAD.

Methods 557 consecutive patients (50.4\% male; median age $55 \mathrm{yrs}$ ) attending rapid access chest pain clinics (RACPC) at two hospitals were risk stratified using NICE criteria. Frequency of admission with suspected angina, diagnosis of $\mathrm{CAD}$ and incidence of major adverse cardiac events (MACE: myocardial infarction (MI), cerebrovascular accident (CVA), emergency revascularisation or cardiac-related death) were compared for all risk categories at 6 months.

Results Of $360 / 557$ patients with "non-anginal" pain, 14 (3.9\%) were subsequently admitted with angina, 34 (9.4\%) were diagnosed with CAD, $3(0.8 \%)$ with MI and $2(0.6 \%)$ with CVA. This group accounted for $36.9 \%$ of all patients diagnosed with $\mathrm{CAD}$ and $38.5 \%$ of all patients with MACE. Of 10/557 patients with atypical/typical anginal pain and a likelihood of $\mathrm{CAD}<10 \%, 1(10 \%)$ was diagnosed with $\mathrm{CAD}$. None were admitted with angina or diagnosed with MACE. This group accounted for $1.1 \%$ of all patients diagnosed with $\mathrm{CAD}$

Conclusions This study suggests one in ten patients routinely excluded from cardiac investigation by the NICE algorithm have $\mathrm{CAD}$ and just over one in a hundred of them have a MACE episode. Although these patients are considered low risk, they account for one third of adverse cardiac events in patients attending RACPC.

\section{REFERENCE}

1. National Institute for Health and Clinical Excellence. CG95 Chest pain of recent onset assessment and diagnosis of recent onset chest pain or discomfort of suspected cardiac origin. NICE, 2010.

\section{ASSESSMENT OF PRE-TEST LIKELIHOOD OF CORONARY ARTERY DISEASE IN PATIENTS WITH CHEST PAIN OF RECENT ONSET}

doi:10.1136/heartjnl-2012-301877b.140

I U Haq, * P C Adams. Royal Victoria Infirmary, Newcastle upon Tyne, UK

Background The NICE guideline for chest pain of recent onset recommends diagnosis of angina based on clinical assessment and estimated likelihood of coronary artery disease (CAD). Pre-test likelihood (PTL) estimates are provided in a simplified table based on the Pryor risk equation. If the PTL of CAD is $<10 \%$ or $>90 \%$, further diagnostic testing is not required. If $10 \%-29 \%$ cardiac CT should be offered, if $30 \%-60 \%$ functional imaging, and if $61 \%-90 \%$ coronary angiography. We compared risk estimation methods to determine how much use of the table in an uncritical manner vs use of the full risk equation led to a different referral pattern. We also investigated whether interpolation for risk factor profiles would improve assessment.

Methods Data were collected prospectively for all patients referred to the Rapid Access Chest Pain Clinic, Newcastle upon Tyne, between February 2002 and August 2011. For each patient, PTL of CAD was assessed by three methods: (1) Reference to the NICE table based on chest pain (typical or atypical), age in deciles, sex and risk factors-smoking, hyperlipidaemia and diabetes dichotomised into high and low risk. For high risk, all three risk factors needed to be present; otherwise the patient was assumed low risk. (2) As per method one, but risk estimates were interpolated between low and high risk values in the NICE table according to the number of risk factors. (3) Calculation by the Pryor equation which includes, in addition, age in years, prior MI, ECG $\mathrm{Q}$ waves, and ST/T changes.

Results Out of an initial 7022 patients, 1820 were excluded as they had non-anginal chest pain. This left 5202 patients, 2889 with atypical angina and 2313 with typical angina. The number (\%) of patients in the five different risk groups by the three risk assessment methods is given in Abstract 140 table 1. Use of the risk equation compared to the table led to half as many people in the category $<10 \%$. Fewer patients would be referred for cardiac CT, more for functional imaging and more for invasive coronary angiography. Use of the adapted table correlated with the risk equation better, but there were still discrepancies, and the percentage of patients for coronary angiography would increase overall (Abstract 140 table 1). The NICE table would classify only $39 \%$ of patients with PTL $<10 \%$ correctly, $60.3 \%$ of those with PTL $10 \%-29 \%, 44.9 \%$ of those with PTL $30 \%-60 \%, 62.9 \%$ of those with PTL $61 \%-90 \%$, and $86.3 \%$ of those with PTL $>90 \%$ (Abstract 140 figure 1). The corresponding figures correctly classifying people in the five risk categories using the adapted table were $92.9 \%, 86.4 \%, 65.2 \%, 71.2 \%$ and $85.9 \%$ respectively (Abstract 140 figure 2).

\section{Abstract 140 Table 1}

\begin{tabular}{llrlll}
\hline $\begin{array}{l}\text { Pre-test } \\
\text { likelihood CAD }\end{array}$ & $<\mathbf{1 0 \%}$ & $\mathbf{1 0 \% - 2 9 \%}$ & $\mathbf{3 0 \% - 6 0 \%}$ & $\mathbf{6 1 \% - 9 0 \%}$ & $\mathbf{> 9 0 \%}$ \\
\hline NICE table-n (\%) & $365(7.0)$ & $1247(24.0)$ & $1069(20.5)$ & $1424(27.4)$ & $1097(21.1)$ \\
Modified table-n (\%) & $127(2.4)$ & $795(15.3)$ & $1271(24.4)$ & $1821(35.0)$ & $1188(22.8)$ \\
Pryor risk & $183(3.5)$ & $1002(19.3)$ & $1190(22.9)$ & $1633(31.4)$ & $1194(23.0)$ \\
equation-n (\%) & & & & & \\
\hline
\end{tabular}

